\title{
A DAMAGED GENERALISED POISSON MODEL AND ITS APPLICATION TO REPORTED AND UNREPORTED ACCIDENT COUNTS
}

BY

\author{
DAVID P.M. SCOLLNIK
}

\begin{abstract}
This paper investigates some models in which non-negative observations from a Poisson or generalised Poisson distribution are possibly damaged according to a binomial or quasi-binomial law. The latter case is appropriate when the observations are over-dispersed. Although the extent of the damage is not known, it is assumed that the event of whether or not damage occurred is discernible. The models are particularly suited for certain applications involving accident counts when evidence of certain accidents may be observed even though the accidents themselves may go unreported. Given the number of observed accidents and knowledge as to whether or not some additional accidents have gone unreported, these models may be used to make inferences concerning the actual number of unreported and total number of accidents in the current period, and the numbers of reported, unreported, and/or total accidents in a future period. The models are applied to a real data set giving reported and unreported patient accidents in a large hospital. Both maximum likelihood and Bayesian estimation methods are presented and discussed.
\end{abstract}

\section{KEYWORDS}

Accidents, Bayesian, damage, Markov chain Monte Carlo, generalised Poisson, quasi-binomial, survival distribution, unreported.

\section{INTRODUCTION}

Let $X$ be a non-negative integer-valued random variable, with probability mass function (pmf) $\operatorname{Pr}(X=x)$ This random variable is said to be damaged if $X=$ $Y+Z$, where $Z$ is a non-negative integer-valued unobservable random variable representing the amount of damage and $Y$ is the observable part of $X$. An example commonly used to illustrate this particular situation concerns the number of eggs laid by an insect. Let $X$ denote the total number of such eggs. By the time the eggs are counted it is possible that some of the eggs may be lost or destroyed, so that only the total number of intact and undamaged eggs 
is recorded. If this total is denoted as $Y$, then it is reasonable to call $Y$ the undamaged part of $X$ and $X-Y=Z$ the damaged part of $X$. Many other natural phenomena fit this model framework. For example, insect damage to seeds in plants could result in a known and observed number of undamaged seeds and an unknown number of lost or destroyed seeds. In this paper, the particular interest is on the total number of accidents $X$ when the number of accidents may be decomposed into reported accidents $Y$ and unreported accidents $Z$ (e.g., automobile guardrail collisions that are reported to the police, and those that are not).

Damage models of the sort described in this paper may be of value to an insurer whenever it appears that some accidents are going unreported. For example, consider an aging oil refinery. Many small fires and breakdowns may be going unreported, in order to avoid unnecessary attention from government agencies and environmental protection groups. The insurer may be primarily interested in the number of reported accidents in future periods that will result in insurance claims. If some accidents are going unreported, evidence of this fact is available (either directly or from secondary sources such as a trade union or employee association's newsletter), and some knowledge of the reporting mechanism (i.e., the survival distribution as it is defined in the next paragraph) can be introduced into the modelling framework (particularly but not necessarily through a Bayesian analysis), then better estimates of future reported accident counts should result. Unreported accidents may also suggest an accumulation of unsafe working practices, and an accumulation of unsafe working practices may lead to more numerous (and possibly more serious) reported accidents in future periods. This would appear to be knowledge that an insurer should want to include in its loss prediction models.

Suppose $X=Y+Z$ as before. Given $X=x$, the conditional distribution $\operatorname{Pr}(Y=y \mid X=x)$ is known as the survival distribution in the damage model literature. Given the survival distribution, under what circumstances will it characterize the distribution of $X$, and vice versa? A number of papers have investigated this problem, many of which are referenced in Rao and Shanbhag (1982). In particular, Rao and Rubin (1964) proved that when the survival distribution $\operatorname{Pr}(Y=y \mid X=x)$ is binomial $(x, \varphi)$ (i.e., so that every component of $X$ is independently and equally likely to be undamaged with fixed probability $\varphi$ ), the random variable $Y$ is such that

$$
\operatorname{Pr}(Y=y)=\operatorname{Pr}(Y=y \mid X=Y)=\operatorname{Pr}(Y=y \mid X>Y)
$$

(NB, the conditional distribution of $Y$, given no damage, is the same as the marginal distribution of the undamaged part $Y$ of $X$ ) if and only if $X$ has a Poisson distribution. Furthermore, if $X$ is a Poisson random variable with parameter $\theta$, then $Y$ and $Z$ are Poisson variables with parameters $\varphi \theta$ and $(1-\varphi) \theta$, respectively, and $Y$ and $Z$ are also independent (Krishnaji, 1974). Srivastava and Srivastava (1970) proved that if $X$ has a Poisson distribution and if (1.1) is satisfied, then the survival distribution must be binomial. 
Charnet and Gokhale (2004) consider the situation in which $X$ is Poisson with parameter $\theta$, with a binomial $(x, \varphi)$ survival distribution determining the number of these observations $Y$ that are undamaged, and $Z$ denoting the remainder (i.e., the number of damaged observations). Charnet and Gokhale (2004, page 261) further suppose that whenever an observation of $Y$ is made on the (possibly damaged) random variable $X$, it is possible to identify whether some damage has occurred, although not the actual amount of said damage. The indicator random variable $D$ is now introduced and defined to take on the value 1 if damage is observed to have taken place, and 0 otherwise. Observe that the event $[D=0]$ is equivalent to the event $[Z=0]$, and $[D=1]$ is equivalent to $[Z>0]$. From the earlier discussion, it follows that $Y$ and $D$ are independent with

$$
\operatorname{Pr}(Y=y)=e^{-\varphi \theta} \frac{(\varphi \theta)^{y}}{y !}
$$

for $y=0,1,2, \ldots$, and

$$
\begin{aligned}
& \operatorname{Pr}(D=0)=\operatorname{Pr}(Z=0)=e^{-(1-\varphi) \theta}, \\
& \operatorname{Pr}(D=1)=\operatorname{Pr}(Z>0)=1-e^{-(1-\varphi) \theta} .
\end{aligned}
$$

Hence,

$$
\operatorname{Pr}(Y=y, D=d)=e^{-\varphi \theta} \frac{(\varphi \theta)^{y}}{y !}\left(1-e^{-(1-\varphi) \theta}\right)^{d}\left(e^{-(1-\varphi) \theta}\right)^{1-d},
$$

for $y=0,1,2, \ldots$, and $d=0,1$

Based on a random sample $\left(y_{i}, d_{i}\right), i=1,2, \ldots, n$, the log-likelihood function $L$ is given by

$$
-n \varphi \theta+n \bar{y} \ln (\varphi \theta)+n \bar{d} \ln \left(1-e^{-(1-\varphi) \theta}\right)-n(1-\bar{d})(1-\varphi) \theta-\sum_{i=1}^{n} \ln \left(y_{i} !\right),
$$

where $\bar{y}$ and $\bar{d}$ are the sample means of the $y_{i}$ and $d_{i}$ values, for $i=1,2, \ldots, n$, respectively. Hence, the likelihood equations are given by

$$
\frac{\partial L}{\partial \theta}=-n \varphi+\frac{n \bar{y}}{\theta}+\frac{n \bar{d}(1-\varphi)}{e^{(1-\varphi) \theta}-1}-n(1-\bar{d})(1-\varphi)=0
$$

and

$$
\frac{\partial L}{\partial \varphi}=-n \theta+\frac{n \bar{y}}{\varphi}-\frac{n \bar{d} \theta}{e^{(1-\varphi) \theta}-1}+n(1-\bar{d}) \theta=0 .
$$

These likelihood equations can be solved explicitly and the resulting maximum likelihood estimators are

$$
\hat{\theta}=\bar{y}-\ln (1-\bar{d})
$$


and

$$
\hat{\varphi}=\frac{\bar{y}}{\hat{\theta}}=\frac{\bar{y}}{\bar{y}-\ln (1-\bar{d})} .
$$

Charnet and Gokhale (2004) study the properties and behaviour of these maximum likelihood estimators. They illustrate their results using a data set of reported monthly accident counts for an energy company in Sao Paulo, Brazil, artificially adjusted in order to simulate unreported accidents.

This paper will introduce a more general case of the damage model described above for use when the counts have unequal exposures associated with them, introduce an alternative damage model based on a generalised Poisson distribution and a quasi-binomial distribution that may be more appropriate when the counts are overdispersed, and consider how to implement the maximum likelihood estimation and the Bayesian analysis of these models. Given the number of observed accidents and knowledge as to whether or not some additional accidents have gone unreported, these models may be used to make inferences concerning the actual number of unreported and total number of accidents in the current period, and the numbers of reported, unreported, and/or total accidents in a future period. The results are illustrated using a real data set describing reported and unreported patient accidents in a large acute study hospital. Henceforth, at least for the most part, this paper will discuss these damage models in the context of reported and unreported accidents. However, it should be understood that the models are more generally applicable.

\section{THE CASE OF UNEQUAL EXPOSURES}

The case of unequal exposures is easily addressed. Let the total number of accidents $X$ have a Poisson distribution with mean $w \theta$. The parameter $\theta$ is the mean accident rate per unit of exposure and $w$ denotes the units of exposure associated with $X$ (e.g., number of employees, number of hours worked, paid premium, etc.). Suppose that $X=Y+Z$, with $Y$ and $Z$ denoting the number of reported and unreported accidents, respectively. Assume that a particular accident is independently and equally likely to be reported with fixed probability $\varphi$ so that the survival distribution $\operatorname{Pr}(Y=y \mid X=x)$ is binomial $(x, \varphi)$. From the discussion in the previous section, it follows that $Y$ and $Z$ are Poisson variables with parameters $w \varphi \theta$ and $w(1-\varphi) \theta$, respectively, and $Y$ and $Z$ are also independent.

Suppose that whenever an observation of $Y$ is made on $X$, it is possible to identify whether some accidents have gone unreported. The actual number of unreported accidents is not known. Let the indicator random variable $D$ take on the value 1 if an unreported accident is observed to have taken place, and 0 otherwise. The event $[D=0]$ is equivalent to the event $[Z=0]$, and $[D=1]$ is equivalent to $[Z>0]$. It follows that $Y$ and $D$ are independent. Further, 


$$
\operatorname{Pr}(Y=y, D=d) e^{-w \varphi \theta} \frac{(w \varphi \theta)^{y}}{y !}\left(1-e^{-w(1-\varphi) \theta}\right)^{d}\left(e^{-w(1-\varphi) \theta}\right)^{1-d},
$$

for $y=0,1,2, \ldots$, and $d=0,1$.

Based on a random sample $\left(y_{i}, d_{i}\right), i=1,2, \ldots, n$, with associated exposures $w_{i}, i=1,2, \ldots, n$, the log-likelihood function $L$ is given by

$$
\begin{aligned}
& -\varphi \theta n \bar{w}+n \bar{y} \ln (\varphi \theta)+\sum_{i=1}^{n} d_{i} \ln \left(1-e^{-w_{i}(1-\varphi) \theta}\right) \\
& -(1-\varphi) \theta \sum_{i=1}^{n}\left(1-d_{i}\right) w_{i}+\sum_{i=1}^{n} y_{i} \ln \left(w_{i}\right)-\sum_{i=1}^{n} \ln \left(y_{i} !\right),
\end{aligned}
$$

where $\bar{y}$ is the sample mean of the $y_{i}$ values, for $i=1,2, \ldots, n$, and $\bar{w}=\sum_{i=1}^{n} w_{i} / n$ is the average exposure. The resulting likelihood equations can be reduced to

$$
\varphi=\frac{\bar{y}}{\theta \bar{w}}
$$

and

$$
\sum_{i=1}^{n}\left(1-d_{i}\right) w_{i}=\sum_{i=1}^{n} \frac{d_{i} w_{i}}{e^{w_{i}(1-\varphi) \theta}-1}
$$

The solution to these equations defines the maximum likelihood estimators $\hat{\theta}$ and $\hat{\varphi}$. It is evident that they reduce to (1.2) and (1.3) in the special case that $w_{i}=1$, for $i=1,2, \ldots, n$.

It should be noted that when all of the $d_{i}$ values are equal to 0 , i.e., when no accidents go unreported, the maximum likelihood estimate of $\varphi$ is one, and the maximum likelihood estimate of $\theta$ is the same as if we had observed a sample of the random variable $X$, i.e., $\theta=\bar{x} / \bar{w}$ (cf. Charnet and Gokhale, 2004).

\section{The generalised Poisson AND QUASI-BINOMial Distributions}

The model described in the previous sections assumes a Poisson distribution for the total number of accidents $X$, and a binomial survival distribution for $Y$ given $X=x$. The Poisson distribution has its theoretical mean equal to its variance, and may not be appropriate if the sample variance of the observations greatly exceeds the sample mean (i.e., if the data is over-dispersed). It is well known that many real life data sets are over-dispersed and many of the discrete distributions used for accident counts and insurance claim frequencies share this property (e.g., see Klugman, Panjer, and Willmot, 2004). The generalised Poisson (also known as the Lagrangian Poisson) distribution (GPD) is an overdispersed generalisation of the basic Poisson model that has found application in many different contexts (Consul, 1989), including those involving accident 
counts and insurance claim frequencies as in Ambagaspitiya (1998), Ambagaspitiya and Balakrishnan (1994), Famoye, Wulu, and Singh (2004), Ntzoufras, Katsis, and Karlis (2005), Scollnik (1995a, 1998), and Vernic (1997, 2000).

The GPD is a two-parameter discrete distribution with its pmf given by

$$
\operatorname{Pr}(X=x)=\theta(\theta+x \lambda)^{x-1} \frac{e^{-\theta-x \lambda}}{y !},
$$

for $x=0,1,2, \ldots$, and zero otherwise, with $\theta>0$ and $0 \leq \lambda<1$ (Consul, 1989). This distribution is such that

$$
E(X)=\frac{\theta}{1-\lambda} \text { and } \operatorname{Var}(X)=\frac{\theta}{(1-\lambda)^{3}},
$$

so its variance is always larger than or equal to its mean. The GPD reduces to the standard Poisson distribution with mean $\theta$ when $\lambda=0$. Some authors allow $\lambda<0$ in order to permit cases in which the variance is smaller than the mean (e.g., Ambagaspitiya and Balakrishnan, 1994; Famoye and Consul, 1995; Vernic, 1997). In this case, the support of $X$ will need to be truncated and, strictly speaking, the pmf rescaled (cf. Consul and Shoukri, 1985; Consul and Famoye, 1989). It should be noted that the expressions given above for the mean and variance, along with virtually all of the other theoretical results that have been obtained for the GPD, are only valid when $0 \leq \lambda<1$, although this has not always been made clear in the literature (e.g., Alzaid and Al-Osh, 1993; Famoye and Consul, 1995). Henze and Klar (1995, page 1877) and Scollnik (1998, page 137) discuss this issue in more detail. In this paper, it will be assumed that $0 \leq \lambda<1$.

Consul (1975a, 1975b, 1989) developed a number of characterizations of the GPD in the context of a damage model. Several of these involve a quasi-binomial distribution (QBD) (Consul and Mittal, 1975; Consul, 1989; cf. Consul, 1974). The QBD in question is a discrete distribution with its pmf given by

$$
\operatorname{Pr}(X=x)=\left(\begin{array}{l}
n \\
x
\end{array}\right) \frac{\varphi(1-\varphi)}{1+n \xi}\left(\frac{\varphi+x \xi}{1+n \xi}\right)^{x-1}\left(\frac{(1-\varphi)+(n-x) \xi}{1+n \xi}\right)^{n-x-1},
$$

for $x=0,1, \ldots, n$, and zero otherwise, with $0 \leq \varphi \leq 1$, and $0 \leq \xi \leq 1$. This reduces to the binomial pmf when $\xi=0$. To be very precise on this matter, (3.2) is the pmf associated with a QBD of type II (Consul and Mittal, 1975; Consul, 1989, pages 27-28). The mean of this distribution is $n \varphi$. Although a simple expression for its variance does not exist, it is simple enough to calculate given the finite support and discrete nature of this distribution. This distribution will have positive or negative extra-binomial variation depending upon the values of $\varphi$ and $\xi$. Also, note that Consul and Mittal (1975, page 71) have derived a complicated formula, along with upper and lower bounds, for the variance.

Assume the same set-up for the damage model as before. That is, let $X$ be a non-negative integer-valued random with $X=Y+Z$, where $Z$ is a non-negative 
integer-valued unobservable random variable representing the amount of damage and $Y$ is the observable part of $X$. If the survival distribution $\operatorname{Pr}(Y=y \mid X=x)$ is a QBD with pmf

$$
\operatorname{Pr}(Y=y \mid X=x)=\left(\begin{array}{l}
x \\
y
\end{array}\right) \frac{\varphi(1-\varphi)}{1+x \xi}\left(\frac{\varphi+y \xi}{1+x \xi}\right)^{y-1}\left(\frac{(1-\varphi)+(x-y) \xi}{1+x \xi}\right)^{x-y-1},
$$

for $y=0,1, \ldots, x$, and zero otherwise, with $0 \leq \varphi \leq 1$, and $0 \leq \xi \leq 1$, then the random variable $Y$ is such that

$$
\operatorname{Pr}(Y=y) \operatorname{Pr}(Y=y \mid X=Y) \operatorname{Pr}(Y=y \mid X>Y)
$$

if and only if $X$ has a GPD with parameters $\theta$ and $\lambda=\xi \theta$, that is,

$$
\operatorname{Pr}(X=x)=\theta(\theta+x \xi \theta)^{x-1} \frac{e^{-\theta-x \xi \theta}}{y !},
$$

for $x=0,1,2, \ldots$, and zero otherwise, with $\theta>0$ and $0 \leq \xi \theta<1$ (Consul, 1975a; Consul, 1989). Furthermore, if $X$ has a GPD with parameters $\theta$ and $\xi \theta$, then $Y$ has a GPD with parameters $\varphi \theta$ and $\xi \theta, Z$ has a GPD with parameters $(1-\varphi) \theta$ and $\xi \theta$, and $Y$ and $Z$ are independent (Consul 1975a). Finally, if $X$ has a GPD with parameters $\theta$ and $\xi \theta$ and condition (3.4) is satisfied, then the survival distribution must be a QBD (Consul 1975a; Consul 1989).

\section{THE NATURE OF REPORTED AND UNREPORTED ACCIDENTS AND THE QBD}

It goes without saying that not all accidents are reported. For example, consider the case of traffic accidents. Morocoima-Black, Chavarra, and Cruse (2001) note that not all traffic accidents are reported to police. In particular, they observe that many jurisdictions have thresholds of property damage below which crashes are not investigated. In other instances, motorists may not report crashes for fear of increased insurance rates. Also, crashes on private property may go unreported. There are many other reasons why accidents, traffic-related or otherwise, may go unreported. It may be that an individual experiencing an accident may not know to whom the accident should be reported. Or the individual may not even realise that such an accident should be reported in the first place.

A binomial survival distribution for the number of reported accidents assumes that each accident is independently and equally likely to be reported. However, this is often not the nature of the reporting process for accidents. For example, various safety incentive programs, or schemes, have been identified by which employers may attempt to hide their safety problems in order to reduce costs and escape the notice of regulatory authorities. One such program is a 
safety bingo or raffle that awards prizes to employees, but only employees with no reported accidents are allowed to participate in the prize drawing. Another scheme goes by the name of "Safety Monopoly". In this, management assigns workers to teams and then each week each team advances one step towards winning prize money. But if one member on a team reports an accident or injury, then the entire team is penalized for that week by not moving ahead a step. Schemes such as these may contravene the assumptions underlying a binomial survival distribution for reported accidents.

It has been argued that living beings and leaders of groups of living beings may target a certain number of successful events, act and plan their efforts accordingly, and consequently adjust the probability of success proportionately to the number of successes aimed at (Consul, 1975a, page 556). Safety incentive programs of the sort described in the paragraph above could be an example of this sort of behaviour, as they may encourage groups to target zero reported accidents. On the other hand, one can imagine scenarios in which groups of workers are encouraged by their union or workers association to fully report all accidents; otherwise, unsafe workplace conditions may go unidentified and unremedied. Consul (1975a, pages 555-556) notes that the GPD and QBD share a property in that the probability of success of an event varies proportionately to the number of successes targeted, as evidenced by (3.1) and (3.3). For this reason, it is useful to explore a damage model based on the GPD and QBD in the accident reporting context. Of course, it is also advantageous that the GPD permits over-dispersion, and that the QBD permits positive or negative extra-binomial variation, as mentioned previously.

\section{AN OVERDISPERSED GENERALISED POISSON DAMAGE MODEL}

Based on the discussion in the sections above, it is theoretically legitimate and contextually reasonable for us to base a damage model for reported and unreported accidents on the GPD, with a QBD survival distribution. Taking this to be the case, suppose the total number of accidents in a period associated with one unit of exposure has a GPD with parameters $\theta, \theta>0$, and $\lambda, 0 \leq \lambda<1$. If $X$ denotes the number of accidents in a period associated with $w$ units of exposure, then $X$ has a GPD with parameters $w \theta$ and $\lambda$, based on the convolution property of the GPD (Consul, 1989, page 14). As before, let $X=Y+Z$, with $Y$ and $Z$ denoting the number of reported and unreported accidents, respectively. Under the assumed damage model, the random variable $Y$ has a GPD with parameters $w \varphi \theta$ and $\lambda, Z$ has a GPD with parameters $w(1-\varphi) \theta$ and $\lambda$, and $Y$ and $Z$ are independent. Also, the survival distribution $\operatorname{Pr}(Y=y \mid X=x)$ is a QBD with parameters $\varphi$ and $\xi$ and pmf as given by (3.3) (NB, $\lambda=\xi \theta$ as before).

Once again, assume that it is possible to identify whether some accidents have gone unreported whenever an observation of $Y$ is made on $X$. The actual number of unreported accidents is not known. Let the indicator random variable $D$ take on the value 1 if an unreported accident is observed to have taken 
place, and 0 otherwise. The event $[D=0]$ is equivalent to the event $[Z=0]$, and $[D=1]$ is equivalent to $[Z>0]$. It follows that the random variables $Y$ and $D$ are independent. Further,

$$
\begin{aligned}
\operatorname{Pr}(Y=y, D=d) & =w \varphi \theta(w \varphi \theta+y \lambda)^{y-1} \frac{e^{-w \varphi \theta-y \lambda}}{y !} \\
& \times\left(1-e^{-w(1-\varphi) \theta}\right)^{d}\left(e^{-w(1-\varphi) \theta}\right)^{1-d},
\end{aligned}
$$

for $y=0,1,2, \ldots$, and $d=0,1$.

\subsection{Maximum likelihood estimates}

Based on a random sample $\left(y_{i}, d_{i}\right), i=1,2, \ldots, n$, with associated exposures $w_{i}$, $i=1,2, \ldots, n$, the log-likelihood function $L$ is given by

$$
\begin{aligned}
& -\varphi \theta n \bar{w}-\lambda n \bar{y}+n \ln (\varphi \theta)+\sum_{i=1}^{n}\left(y_{i}-1\right) \ln \left(w_{i} \varphi \theta+y_{i} \lambda\right) \\
& +\sum_{i=1}^{n} d_{i} \ln \left(1-e^{-w_{i}(1-\varphi) \theta}\right)-(1-\varphi) \theta \sum_{i=1}^{n}\left(1-d_{i}\right) w_{i} \\
& +\sum_{i=1}^{n} \ln \left(w_{i}\right)-\sum_{i=1}^{n} \ln \left(y_{i} !\right)
\end{aligned}
$$

where $\bar{y}$ is the sample mean of the $y_{i}$ values, for $i=1,2, \ldots, n$, and $\bar{w}=\sum_{i=1}^{n} w_{i} / n$ is the average exposure. The resulting likelihood equations are not readily simplified and cannot be explicitly solved. However, the maximum likelihood estimates, their standard errors, and confidence intervals for the model parameters $\varphi, \theta$, and $\lambda$ may be obtained through direct numerical means. Klugman, Panjer, and Willmot (2004, Sections 12.2 and 12.3, and Appendix F) describe some numerical approaches used in maximum likelihood estimation. The necessary values may also be obtained with the assistance of standard statistical software such as the 'stats4' or 'Bhat' packages of functions for use in R. (R is a language and environment for statistical computing and graphics. See www. $\mathrm{r}$-project.org and cran.r-project.org for the $\mathrm{R}$ software and related information, including downloadable manuals. In particular, see the manual by Venables, Smith, and the R Development Core Team (2005) for an introduction to R.)

\subsection{Bayesian approach}

A Bayesian method of statistical inference interprets all unknown model parameters as random variables in their own right. Depending upon one's point of view, this interpretation may be an honest one or it may be more akin to an operational assumption. In either case, the mathematics of the analysis remain 
the same. A Bayesian analysis determines the conditional distribution of any unknown model parameters and/or future observations, given the observed data and the available prior knowledge. The Bayesian approach has been recommended by many authors for a number of reasons. It facilitates common-sense interpretations of statistical conclusions such as confidence intervals on parameters and P-values, and may improve on the properties of classical estimators in terms of the precision of estimates (Gelman, Carlin, Stern, and Rubin, 2004, page 3; Congdon, 2001, pages 1-2). Bayesian methods automatically deal with nuisance parameters (i.e., parameters in the model that are not of interest), unlike frequentist methods. By its nature, Bayesian statistics is predictive and automatically incorporates parameter uncertainty into the predictions; again, unlike conventional frequentist procedures. For more information on these latter points, refer to Bolstad (2004, page 7).

Makov, Smith, and Liu (1996) observed that 'statistical methods with a Bayesian flavour, in particular credibility theory, have long been used in the insurance industry as part of the process of estimating risks and setting premiums'. In recent years, many authors have used Bayesian methods to implement actuarial models. Examples of this include Cairns (2000), Ntzoufras and Dellaportas (2002), Ntzoufras, Katsis, and Karlis (2005), Rosenberg and Young (1999), Scollnik (1998, 2001), and Verrall (2004). Makov (2001), and the discussants of his paper, detail many other applications of Bayesian methods in actuarial science.

If a Bayesian analysis of our damage model is sought, then a prior distribution must be set forth for the unknown parameters in the model. However, rather than expressing the prior distribution in terms of $\theta, \lambda$, and $\varphi$, it may be more convenient to elicit and describe the prior in terms of easily interpretable functions of these parameters instead. For example, let

$$
\begin{gathered}
\mu=E(Y)=\frac{\varphi \theta}{1-\lambda}, \\
\delta=\frac{\operatorname{Var}(Y)}{E(Y)}=\frac{1}{(1-\lambda)^{2}},
\end{gathered}
$$

and

$$
\eta=\operatorname{Pr}(Z=0)=e^{-(1-\varphi) \theta} .
$$

Observe that $\mu>0, \delta>0$, and $0 \leq \eta \leq 1$. Here, $\mu$ is the mean number of reported accidents, $\delta$ is the index of dispersion (i.e., the ratio of the variance to the mean) associated with the number of reported accidents, and $\eta$ is the probability of there being zero unreported accidents, all assuming one unit of exposure, and given the model parameters. Bearing in mind that $\theta>0,0 \leq \lambda<1$, and $0 \leq \varphi \leq 1$, the inverse relations are given by

$$
\theta=\frac{\mu}{\sqrt{\delta}}-\ln (\eta),
$$




$$
\lambda=1-\frac{1}{\sqrt{\delta}},
$$

and

$$
\varphi=\frac{\mu}{\mu-\sqrt{\delta} \ln (\eta)}
$$

Presumably, an experienced practitioner familiar with the context of the problem under investigation will be able to describe one or more of the parameters $\mu, \delta$, and $\eta$ in terms of a moderately informative prior distribution on the basis of his or her past experience. If not, it may be possible to obtain prior information on the basis of a pilot study or an industry wide survey, depending on the nature of the particular problem at hand. As $\mu$ is a positive parameter, it might be assigned a gamma prior distribution. As the parameter $\eta$ represents a probability, it might be assigned a beta distribution. The parameters for these prior distributions should be set in accordance with the prior information that is available. If significant prior information is simply not available, then relatively spread out versions of these distributions might be adopted in the same manner as in many of the default examples provided with the WinBUGS software (see below). Specifically, $\mu$ might be assigned a gamma $(0.01,0.01)$ prior, and $v$ might be assigned a beta $(1,1)$ (i.e., uniform) prior. Note, Scollnik (1995b) previously examined a variety of priors for use with $\delta$ in the context of the GPD. If the prior is expressed in terms of $\mu, \delta$, and $\eta$, then

$$
p(\theta, \lambda, \varphi)=p(\mu, \delta, \eta)\left|\frac{\partial(\mu, \delta, \eta)}{\partial(\theta, \lambda, \varphi)}\right|,
$$

where

$$
\left|\frac{\partial(\mu, \delta, \eta)}{\partial(\theta, \lambda, \varphi)}\right|=\frac{2 \theta e^{-(1-\varphi) \theta}}{(1-\lambda)^{4}}
$$

When $\lambda=0$ (i.e., in the case of the standard Poisson damage model), the corresponding result is

$$
p(\theta, \varphi)=p(\mu, \eta)\left|\frac{\partial(\mu, \eta)}{\partial(\theta, \varphi)}\right|
$$

where

$$
\left|\frac{\partial(\mu, \eta)}{\partial(\theta, \varphi)}\right|=\theta e^{-(1-\varphi) \theta} .
$$

The Bayesian analysis may be implemented using a Markov chain Monte Carlo (MCMC) method as described in Gelman et alia (2004). The Bayesian numerical example described in the next section was implemented using the software package WinBUGS (Spiegelhalter, Thomas, Best, and Gilks, 1996; 
Spiegelhalter, Thomas, Best, and Lunn, 2004). WinBUGS is a program for implementing the Bayesian analysis of complex statistical using MCMC techniques. See www.mrc-bsu.cam.ac.uk/bugs and mathstat.helsinki.fi/openbugs for the WinBUGS software and related information, including on-line manuals. Scollnik (2001) describes the use of the WinBUGS software in extensive detail, in the particular context of actuarial modelling. WinBUGS is also described, and illustrated with many examples, in the texts by Gelman et alia (2004), and Congdon (2001, 2003).

\section{NumericAl iLlUSTRATION: ACCIDENTS TO PATIENTS IN HOSPITAL}

Sutton, Standen, and Wallace (1994) investigated the incidence of reported and unreported patient accidents in a large acute study hospital. Data was collected over three widely spaced study days, in the form of a 10-ward sample. The reported and unreported accident counts are reported in Table 1, along with the occupied bed days (OBD) of the study patients. The OBD divided by 1000 serves as the measure of exposure.

In order to illustrate the models developed in this paper, we will ignore the actual observed number of unreported accidents appearing in the fifth column when fitting the models and only make use of the fact whether or not unreported accidents were observed to have taken place. It is important to bear the above in mind throughout the discussion below. This data set is particularly useful from an illustrative perspective as the actual observed unreported accident counts can be used to monitor the predictive performance of the models. However, Sutton et alia (1994, page 48) suggested that the actual observed count of 26 unreported accidents was likely to have been an underestimate of the 'true' frequency of unreported patient accidents, and suggested no less than seven reasons why this should be the case. Some of these reasons hold out the possibility that the numbers of unreported accidents could be vastly understated. For example, unreported accident patients were often multiple accident patients. If a patient had several falls before a doctor's examination, one report form was made to cover the entire lot so that the second and subsequent falls had no accident report forms at all. For this reason and others, the actual numbers of unreported accidents may greatly exceed those given in Table 1.

\subsection{A maximum likelihood based analysis}

To begin with, we apply the basic Poisson damage model (2.1) to the data in Table 1 in the manner described in the paragraph above. The resulting maximum likelihood estimates (and their estimated standard errors) are $\hat{\theta}=12.014$ (2.028) and $\hat{\varphi}=0.687(0.099)$. The $95 \%$ confidence intervals for $\theta$ and $\varphi$ are $(8.720,17.227)$ and $(0.479,0.851)$, respectively. The maximum value of the loglikelihood is $L^{*}=-38.121$. Next, we apply the generalised Poisson damage model (5.1) to the same data. The maximum likelihood estimates (and their estimated 
TABLE 1

DATA ON REPORTED AND UNREPORTED PATIENT ACCIDENTS

\begin{tabular}{ccccc}
\hline $\begin{array}{c}\text { Ward number } \\
\text { number }\end{array}$ & $\begin{array}{c}\text { OBD study } \\
\text { patients }\end{array}$ & $\begin{array}{c}\text { Number of } \\
\text { reported accidents }\end{array}$ & $\begin{array}{c}\text { Unreported } \\
\text { accidents }\end{array}$ & $\begin{array}{c}\text { Number of } \\
\text { unreported accidents }\end{array}$ \\
\hline 1 & 1661 & 8 & Yes & 11 \\
2 & 410 & 10 & Yes & 6 \\
3 & 515 & 2 & Yes & 3 \\
4 & 421 & 0 & Yes & 2 \\
5 & 577 & 6 & Yes & 1 \\
6 & 321 & 8 & Yes & 1 \\
7 & 730 & 3 & Yes & 1 \\
8 & 668 & 0 & Yes & 1 \\
9 & 532 & 0 & No & 0 \\
10 & 100 & 49 & No & 0 \\
\hline Total & 5935 & & & 26 \\
\hline \hline
\end{tabular}

standard errors) are $\hat{\theta}=7.185$ (2.014), $\hat{\lambda}=0.585(0.152)$, and $\hat{\varphi}=0.477(0.138)$. The $95 \%$ confidence intervals for $\theta, \lambda$, and $\varphi$ are $(4.030,12.411),(0.302,0.901)$, and $(0.231,0.731)$, respectively. The maximum value of the log-likelihood is $L^{*}=-29.170$. (All of these estimates, standard errors, confidence intervals and log-likelihood values were obtained numerically in R, making use of the 'stats4' package of functions mentioned earlier.) Note that the value of Akaike's information criterion, equal to $-2 L^{*}+2^{*}$ (number of parameters), is 80.243 for the basic Poisson damage model and 64.338 for the generalised Poisson damage model, thus strongly suggesting that the latter is the preferred model.

Conditional on the maximum likelihood estimates, we can examine the fitted Poisson and generalized Poisson distributions for the number of reported, unreported, and total accidents in all wards, assuming 5935 OBD of study patients as in Table 1. (NB, we proceed on the basis of 'all wards' summaries for clarity and brevity of presentation. The overall results and conclusions are unchanged if we examine the results on a ward by ward basis.) Summary measures for the number of reported, unreported, and total accidents under both fitted models are reported in Table 2 . The probability plots for the fitted models appear in Figures 1 and 2. At first blush it may appear that the generalised Poisson model vastly overstates the possible number of unreported accidents, given that only 26 unreported accidents appear in Table 1 . However, recall from the earlier discussion that the observed count of 26 unreported accidents is very possibly a gross undercount. With this in mind, it appears more likely that the basic Poisson model is understating the possible number of unreported accidents. Thus, it is reassuring that Akaike's information criterion recommended the generalised Poisson damage model over the basic Poisson damage model. 
TABLE 2

ACCIDENT COUNTS FOR THE BASIC AND GENERALISEd Poisson DAMAGE MOdELS FITTED BY MAXIMUM LIKELIHOOD

\begin{tabular}{l|ccccc}
\hline \hline \multirow{2}{*}{$\begin{array}{l}\text { Accidents } \\
\text { (all wards) }\end{array}$} & \multicolumn{5}{|c}{ Summaries for the basic Poisson model } \\
\cline { 2 - 6 } & Mean & SD & $2.5 \%$ & Median & $97.5 \%$ \\
\hline Reported & 48.99 & 7.0 & 36 & 49 & 63 \\
Unreported & 22.32 & 4.72 & 14 & 22 & 32 \\
\hline Total & 71.3 & 8.45 & 55 & 71 & 88 \\
\hline \hline \multirow{2}{*}{ Accidents } & \multicolumn{5}{c}{ Summaries for the generalised Poisson model } \\
\cline { 2 - 6 } (all wards) & Mean & SD & $2.5 \%$ & Median & $97.5 \%$ \\
\hline Reported & 49.01 & 16.87 & 22 & 47 & 87 \\
Unreported & 53.74 & 17.66 & 25 & 52 & 94 \\
\hline Total & 102.76 & 24.43 & 61 & 101 & 156 \\
\hline \hline
\end{tabular}

Maximum likelihood estimates are equivalent to the Bayesian posterior modes that result under locally uniform prior distributions. Hence, the fitted distributions in Table 2 and Figures 1 and 2 are the same as the Bayesian predictive distributions for the number of reported, unreported, and total accidents, conditional on the posterior modes under these flat noninformative priors. We will now consider a Bayesian analysis making use of more informative prior distributions.

\subsection{A Bayesian analysis}

The following illustrative Bayesian analysis of the data will assume that the parameters $\mu, \delta$, and $\eta$, as defined by (5.2), (5.3), and (5.4), respectively, are all a priori independent. The parameter $\mu$ is assigned a gamma prior distribution with mean of 10 and standard deviation equal to 5 . The prior distribution for the parameter $\eta$ is taken to be beta, with mean of 0.25 and standard deviation equal to 0.2 . These two prior distributions are mildly informative, but they still assign significant prior probability to a wide collection of possible values for the parameters $\mu$ and $\eta$. Following Scollnik (1995b), the random quantity $\delta-1$ is assigned an exponential prior distribution with a rate of 0.5 in the case of the generalised Poisson damage model (5.1). Of course, the parameter $\delta$ is simply set equal to 1 (i.e., $\lambda=0$ ) in the case of the basic Poisson damage model (2.1).

The Bayesian analysis was implemented using MCMC in WinBUGS, and the corresponding WinBUGS code is provided in the Appendix. The analysis was run twice, once for the basic Poisson damage model (2.1), and again for 

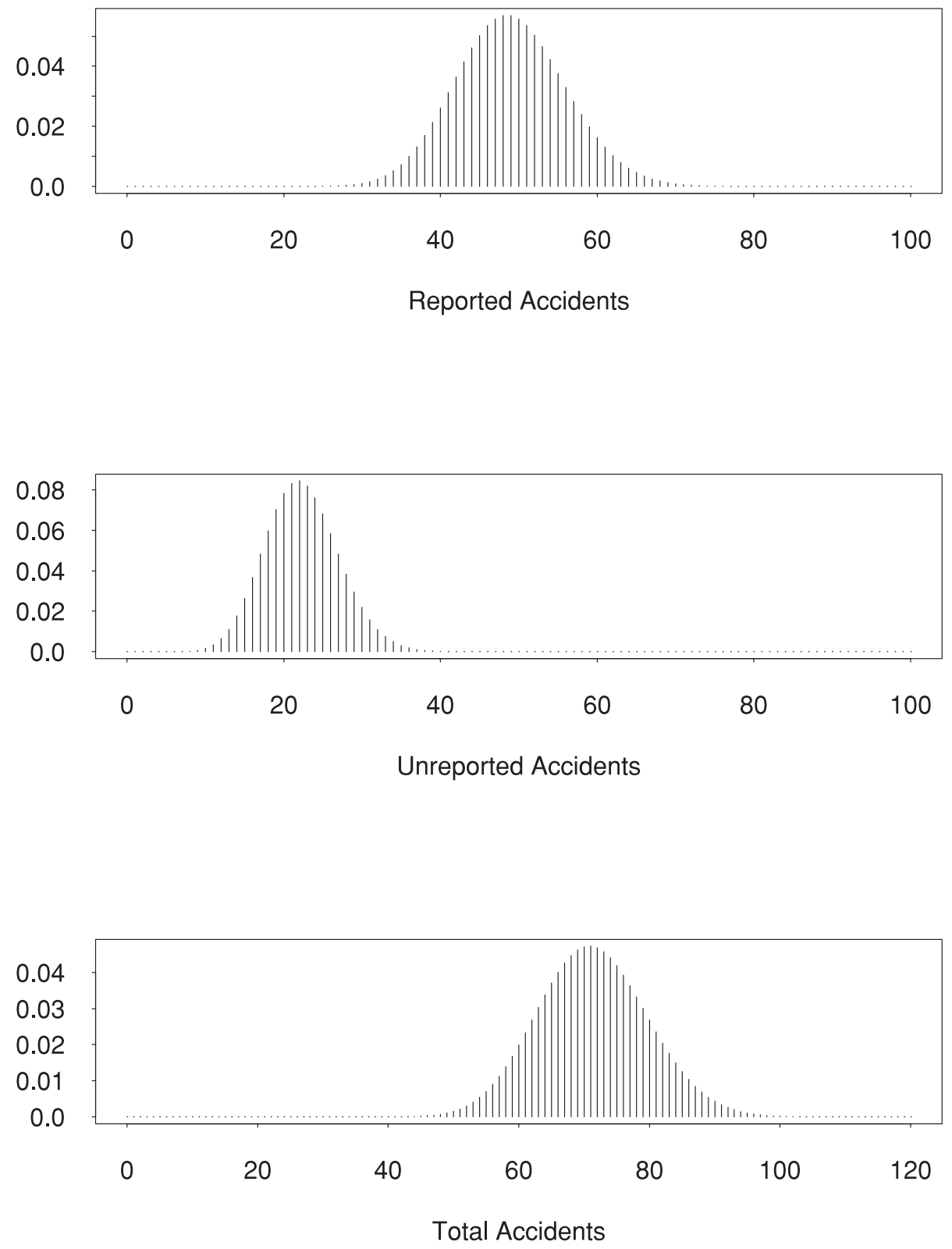

FIGURE 1. Probability functions for the number of reported, unreported, and total accidents according to the basic Poisson model fitted by maximum likelihood. 

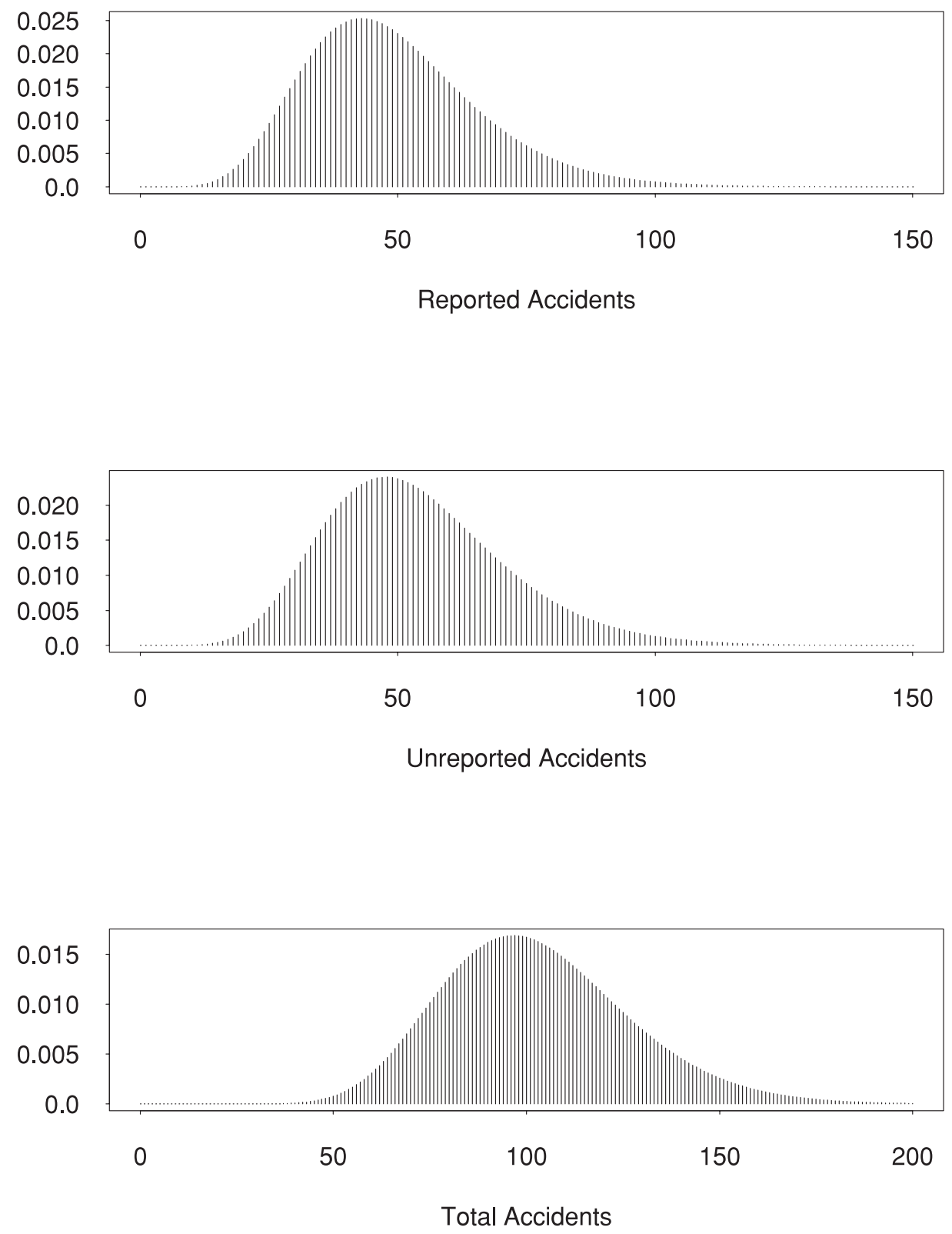

Figure 2. Probability functions for the number of reported, unreported, and total accidents according to the generalised Poisson model fitted by maximum likelihood. 
the generalised Poisson damage model (5.1). The MCMC simulations were monitored for convergence using methods described in Gelman et alia (2004). We also monitored the Gelman-Rubin convergence statistic, as modified by Brooks and Gelman (1998), that is implemented in WinBUGS. Convergence diagnostics in CODA (Convergence Diagnostics and Output Analysis) were also monitored, and no problems with convergence were identified. (CODA is a suite of functions which can be used to analyze the output from WinBUGS. An implementation of CODA is available for use with $\mathrm{R}$, and is available from the BUGS websites mentioned earlier in this paper, or from www-fis.iarc.fr/coda.) In all cases, the results were checked using a number of different initial conditions. Scollnik (2001) extensively details the process of running a model in WinBUGS, monitoring its output, assessing convergence of the simulation, and discusses CODA and the alternative Bayesian Output Analysis Program (BOA) (www. public-health.uiowa.edu/boa) in more depth. Scollnik (2001) may be referenced for more information on these various topics.

Our final inferences are based on four independent chains of simulated values, run for 50,000 iterations apiece, following 10,000 burn-in iterations. Estimated posterior summary statistics (i.e., posterior mean, standard deviation, and $2.5 \%, 50 \%$, and $97.5 \%$ percentiles) for the model parameters are reported in Table 3. The Deviance Information Criterion (DIC), a combined measure of model complexity and fit, was also monitored (Spiegelhalter et alia, 2002). The DIC is 80.819 for the basic Poisson damage model, and is 63.964 for the generalised Poisson damage models. The WinBUGS User manual states that

TABLE 3

ESTIMATED POSTERIOR SUMMARY STATISTICS FOR THE BASIC AND GENERALISED POISSON DAMAGE MODELS

\begin{tabular}{c|ccccc}
\hline \hline \multirow{3}{*}{ Parameter } & \multicolumn{5}{|c}{ Estimates for the basic Poisson model } \\
\cline { 2 - 6 } & Mean & SD & $2.5 \%$ & Median & $97.5 \%$ \\
\hline$\theta$ & 10.26 & 1.242 & 7.975 & 10.21 & 12.85 \\
$\varphi$ & 0.8146 & 0.0418 & 0.7245 & 0.8178 & 0.8873 \\
$\mu$ & 8.368 & 1.156 & 6.262 & 8.315 & 10.79 \\
$\eta$ & 0.1656 & 0.0703 & 0.0543 & 0.157 & 0.3254 \\
\hline \hline \multirow{3}{*}{ Parameter } & \multicolumn{5}{c}{ Estimates for the generalised Poisson model } \\
\cline { 2 - 6 } & Mean & SD & $2.5 \%$ & Median & $97.5 \%$ \\
\hline$\theta$ & 5.849 & 1.178 & 3.788 & 5.767 & 8.382 \\
$\lambda$ & 0.5116 & 0.0991 & 0.2999 & 0.5189 & 0.684 \\
$\varphi$ & 0.669 & 0.0808 & 0.4948 & 0.6753 & 0.8087 \\
$\mu$ & 8.208 & 2.063 & 4.715 & 8.016 & 12.82 \\
$\delta$ & 4.759 & 2.083 & 2.04 & 4.321 & 10.02 \\
$\eta$ & 0.1658 & 0.0709 & 0.0538 & 0.1569 & 0.3262 \\
\hline \hline
\end{tabular}


TABLE 4

ESTIMATED PREDICTIVE ACCIDENT COUNTS FOR THE BASIC AND GENERALISED PoISSON DAMAGE MODELS

\begin{tabular}{l|ccccc}
\hline \hline \multirow{2}{*}{$\begin{array}{l}\text { Accidents } \\
\text { (all wards) }\end{array}$} & \multicolumn{5}{|c}{ Estimates for the basic Poisson model } \\
\cline { 2 - 6 } & Mean & SD & $2.5 \%$ & Median & $97.5 \%$ \\
\hline Reported & 49.67 & 9.847 & 32 & 49 & 70 \\
Unreported & 11.24 & 4.327 & 4 & 11 & 21 \\
\hline Total & 60.92 & 10.76 & 41 & 60 & 83 \\
\hline \hline \multirow{2}{*}{$\begin{array}{c}\text { Accidents } \\
\text { (all wards) }\end{array}$} & Mean & SD & $2.5 \%$ & Median & $97.5 \%$ \\
\cline { 2 - 6 } Reported & 48.82 & 19.8 & 18 & 46 & 95 \\
Unreported & 23.99 & 13.65 & 6 & 21 & 58 \\
\hline Total & 72.81 & 24.84 & 35 & 69 & 132 \\
\hline \hline
\end{tabular}

"the model with the smallest DIC is estimated to be the model that would best predict a replicate dataset of the same structure as that currently observed". Hence, these results suggest that the generalised Poisson damage model (i.e., with $\lambda>0$ ) is to be preferred over the basic Poisson damage model in this particular case.

In addition to the posterior inferences for the model parameters described above, some posterior predictive inferences were also implemented. In particular, posterior predictive draws (as discussed in Gelman et alia, 2004) were obtained for the reported, unreported, and total accidents counts, asuming 5935 total OBD of study patients as in Table 1. Summary statistics are reported in Table 4, and the posterior predictive plots appear in Figures 3 and 4. Recall that there were originally 49 reported and 26 unreported accidents. From Table 4 and Figures 3 and 4, it is apparent that the actual observed count of 49 reported accidents looks to be plausible under both the basic and generalised Poisson models. However, the actual observed count of 26 unreported accidents only appears to be plausible under the generalised Poisson model. Observe that the $95 \%$ predictive probability interval for the number of unreported accidents under the generalised Poisson model is $(6,58)$, suggesting that a number higher than 26 unreported accidents would still be plausible under this model. Under the basic Poisson model, the $95 \%$ predictive probability interval for the number of unreported accidents is $(4,21)$. Again, we recall Sutton et alia's (1994, page 48) suggestion that the actual observed count of 26 unreported accidents was likely to have been an underestimate of the 'true' frequency of unreported patient accidents. All in all, we must conclude that the generalised Poisson model outperforms the basic Poisson model when both are applied to the patient accident data under consideration. 


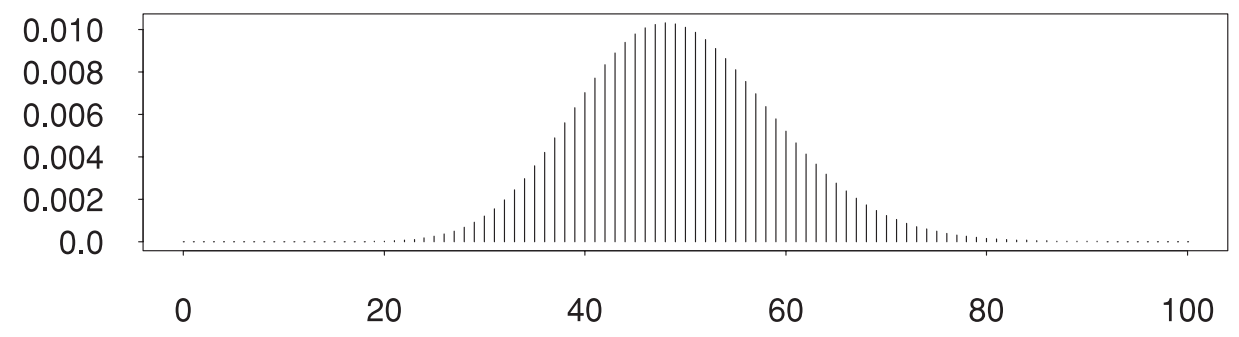

Reported Accidents
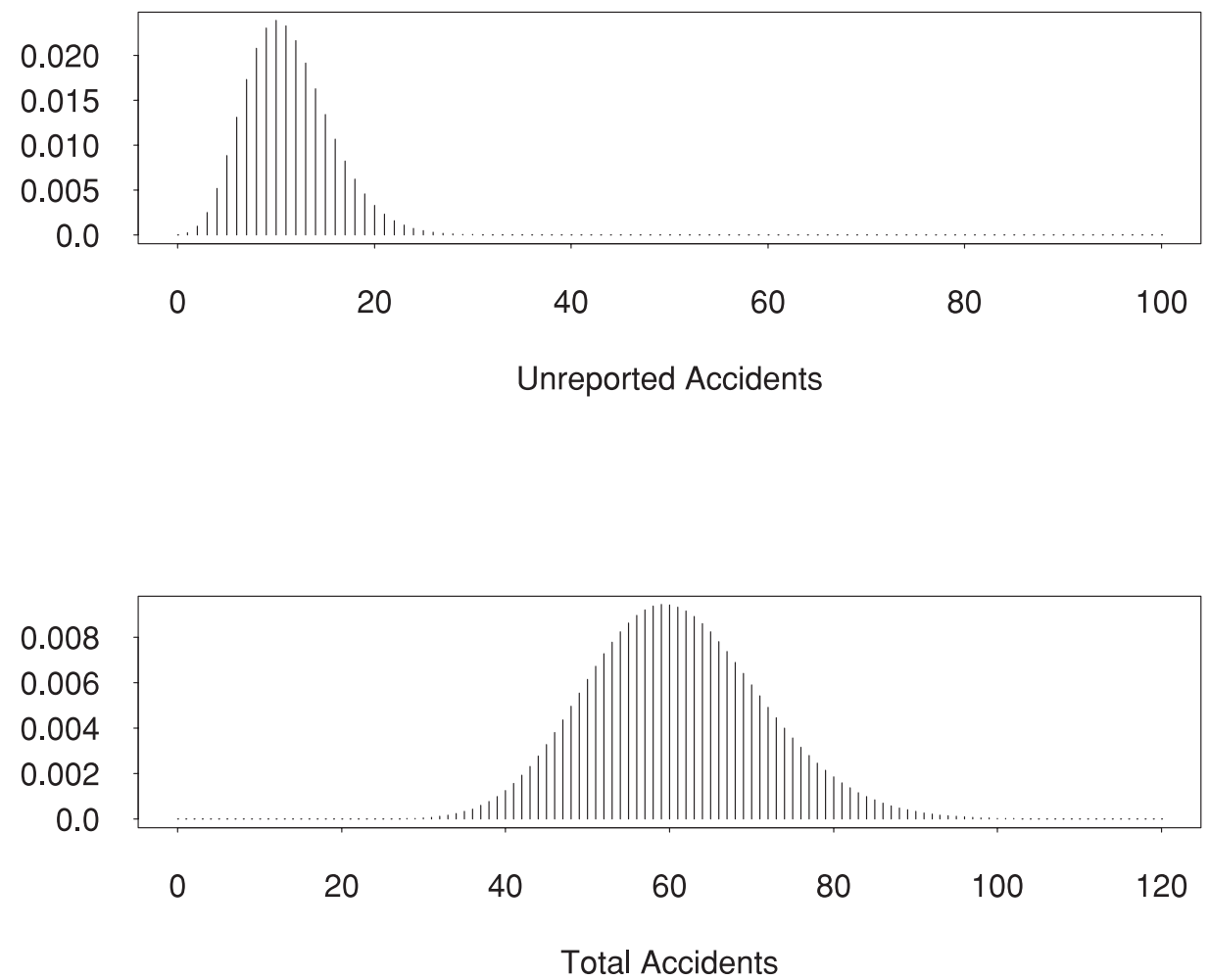

FIGURE 3. Bayesian predictive distribution for the number of reported, unreported and total accidents according to the basic Poisson model. 


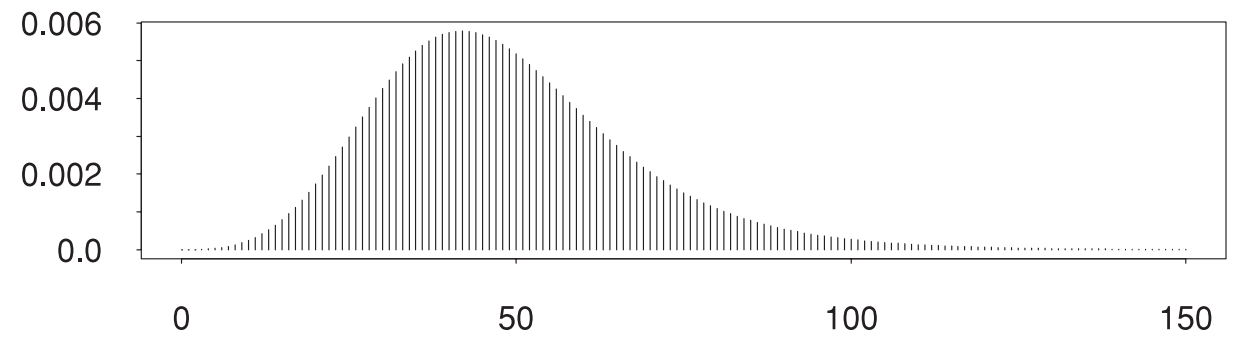

Reported Accidents
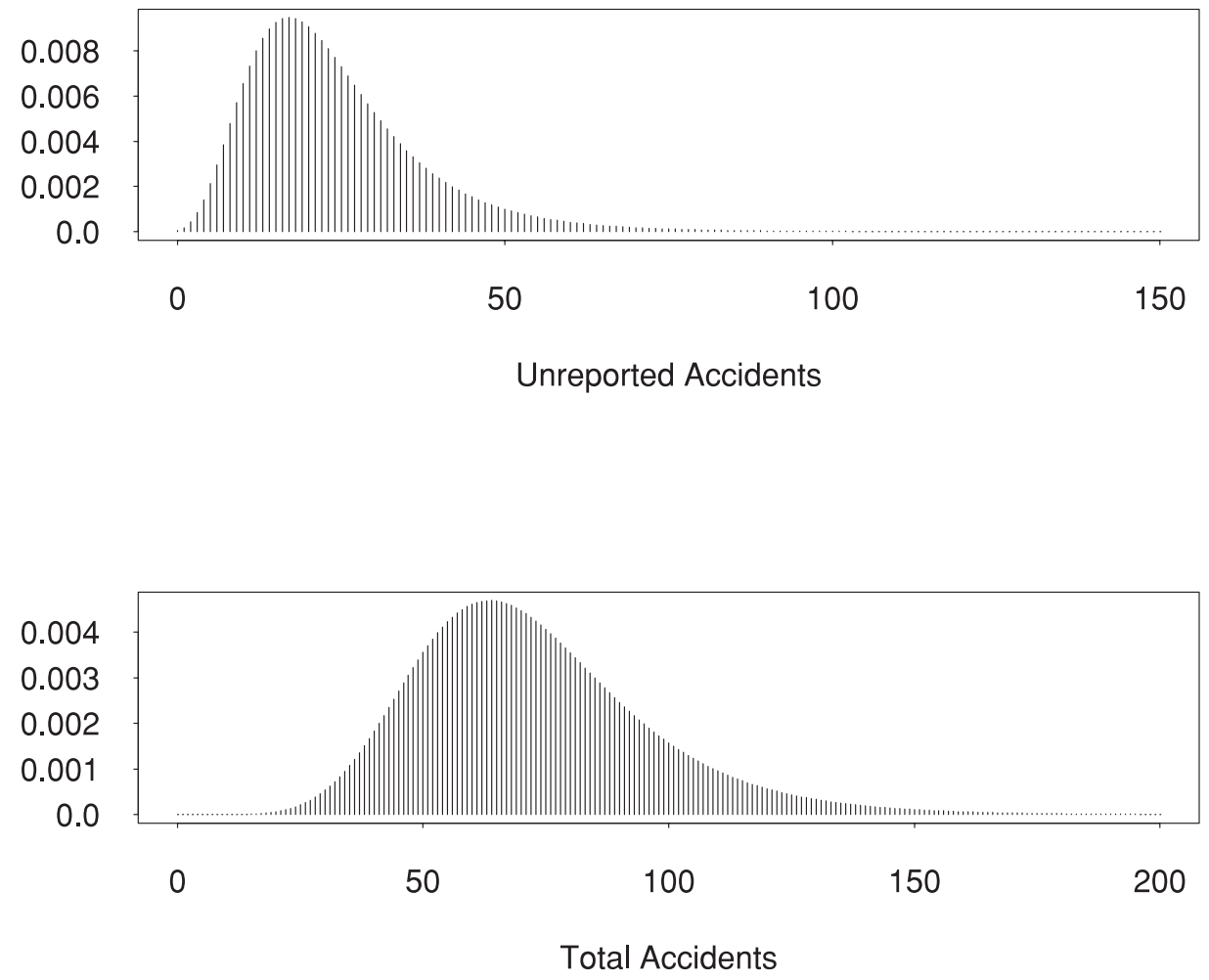

FIGURE 4. Bayesian predictive distribution for the number of reported, unreported and total accidents according to the generalised Poisson model. 
Earlier, we suggested that an experienced practitioner familiar with the context of the accident reporting problem under study should be able to describe one or more of the parameters $\mu, \delta$, and $\eta$ in terms of a moderately informative prior. The informative priors used in our illustrative Bayesian analysis were selected with this in mind. However, the analysis was also repeated using less informative priors. In the various cases we studied, the generalised Poisson damage model consistently won out.

\section{CONCLUding REMARKS}

Readers requiring assistance with the maximum likelihood estimation or with the WinBUGS code described in this paper are encouraged to contact the author. Note that the WinBUGS code is available at a website listed in the Appendix.

As previously mentioned, Scollnik (2001) is an excellent resource for those new to WinBUGS. For those requiring additional background information on Bayesian methods in general, the books by Gelman, Carlin, Stern, and Rubin (2004), and Congdon (2001, 2003) are all highly recommended. These books also emphasise the use of WinBUGS for Bayesian modelling. Klugman, Panjer, and Willmot (2004, Section 12.4) discuss Bayesian methods in the context of various actuarial loss models.

Incidentally, the result that the random variables $Y$ and $Z$ are independent, given the underlying model parameters as in Section 1 and 3, may be surprising and unintuitive, but this is a consequence of the very reasonable assumptions of the binomial (or quasi-binomial) survival distribution in conjunction with the Poisson (or generalised Poisson) distribution for the total number of counts. That these sensible assumptions lead to the independence of $Y$ and $Z$, given the model parameters, is one of the interesting features of the damage models described in this paper. (NB, it should be observed that the Bayesian posterior predictive distributions of future $Y$ and $Z$ values given the observed data will not generally be independent.) If these assumptions really do not appear to be reasonable in the context of a particular problem, then the damage models described in this paper may not be appropriate. Poisson and generalised Poisson models incorporating a reporting mechanism that leads to dependent random variables $Y$ and $Z$ might serve as the focus of a future paper.

\section{ACKNOWLeDGements}

This paper was supported by a grant from the Natural Sciences and Engineering Research Council of Canada (NSERC). The author thanks Dr. Sutton for providing some background information on the patient accidents data used in this paper. The author would also like to thank the anonymous referees for their helpful comments and suggestions. 


\section{APPENDIX \\ WinBUGS CODE FOR THE DAMAGE MODELS}

The following code is available at www.math.ucalgary.ca/ scollnik/abcd/damage

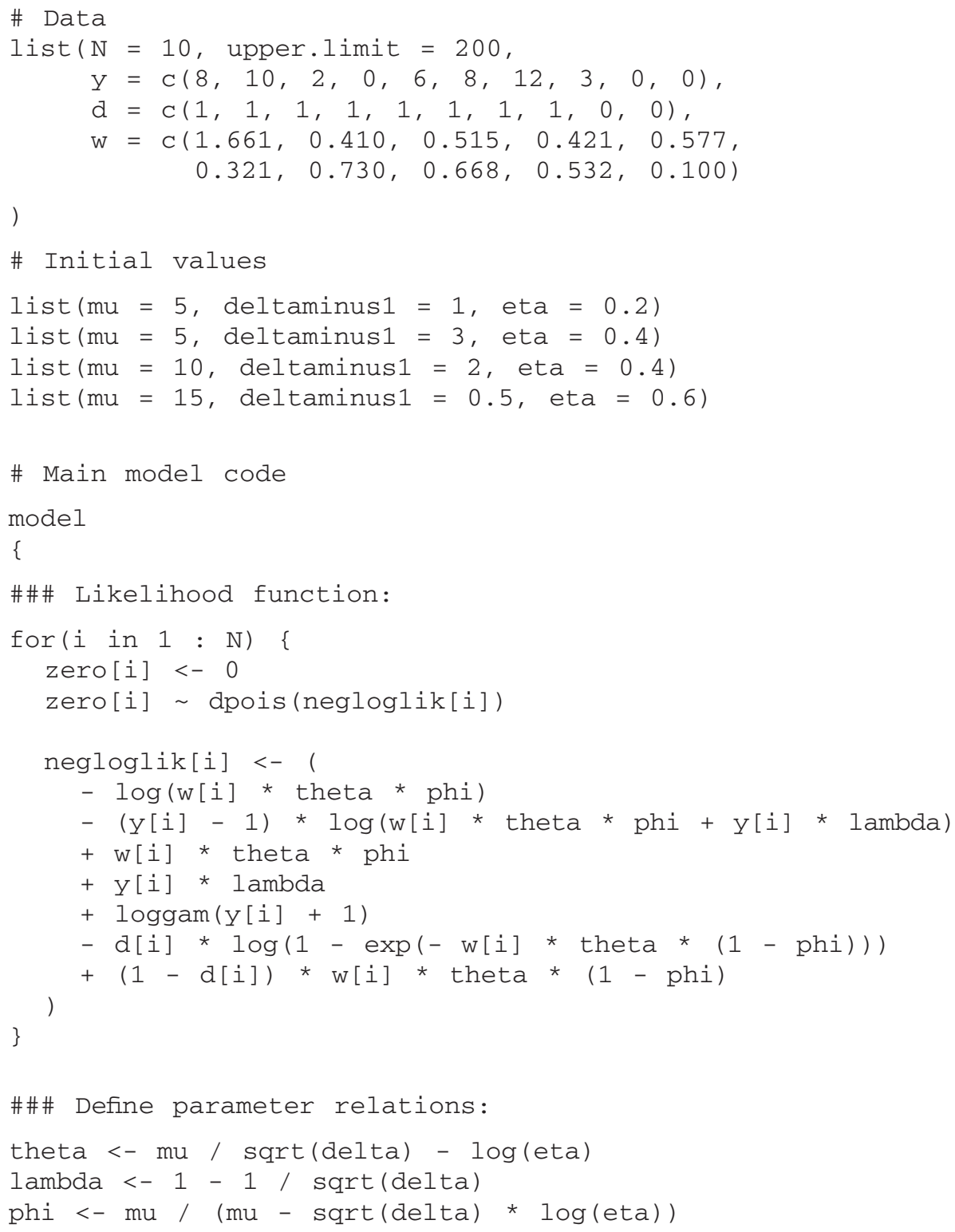




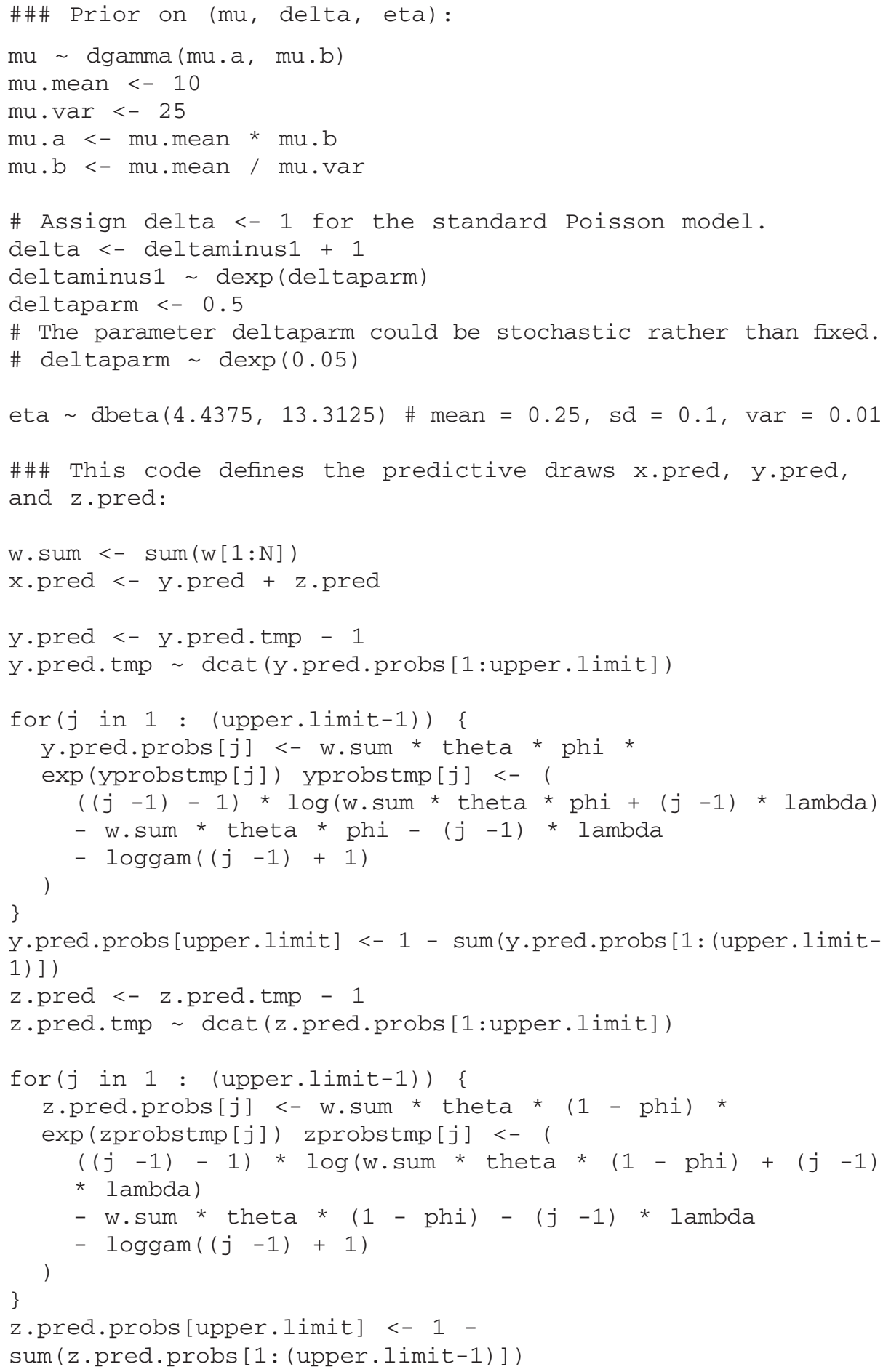




\section{REFERENCES}

Alzaid, A.A. and Al-Osh, M.A. (1993) Some Autoregressive Moving Average Processes with Generalized Poisson Marginal Distributions. Annals of the Institute of Statistical Mathematics 45, 223-232.

AmbagaspitiYA, R.S. (1998) Compound Bivariate Lagrangian Poisson Distributions. Insurance: Mathematics and Economics 23, 21-31.

Ambagaspitiya, R.S. and Balakrishnan, N. (1994) On the Compound Generalized Poisson Distributions. ASTIN Bulletin 24, 255-263.

Bolstad, W.M. (2004) Introduction to Bayesian Statistics. Hoboken: John Wiley \& Sons, Inc.

Brooks, S.P. and Gelman, A. (1998) General Methods for Monitoring Convergence of Iterative Simulations. Journal of Computational and Graphical Statistics 7, 434-455.

CAIRns, A.J.G. (2000) A Discussion of Parameter and Model Uncertainty in Insurance. Insurance: Mathematics and Economics 27, 313-330.

Charnet, R. and Gokhale, D.V. (2004) Statistical Inference for Damaged Poisson Distribution. Communications in Statistics: Simulation and Computation 33, 259-269.

Congdon, P. (2001) Bayesian Statistical Modelling. Chichester: John Wiley \& Sons Ltd.

Congdon, P. (2003) Applied Bayesian Modelling. Chichester: John Wiley \& Sons Ltd.

Consul, P.C. (1974) A Simple Urn Model Dependent Upon Predetermined Strategy. Sankhya, Series B 36, 391-399.

Consul, P.C. (1975a) On a Characterization of Lagrangian Poisson and Quasi-Binomial Distributions. Communications in Statistics 4(6), 555-563.

Consul, P.C. (1975b) Some New Characterizations of Discrete Lagrangian Distributions. Statistical Distributions in Scientific Work, Volume 3, pages 279-290. Patil, G.B., et alia (editors). Hingham: D. Reidel Publishing Co.

Consul, P.C. (1989) Generalized Poisson Distributions: Properties and Applications. New York: Marcel Dekker Inc.

Consul, P.C. (1990) On Some Properties and Applications of Quasi-Binomial Distribution. Communications in Statistics: Theory and Methods 19(2), 477-504.

Consul, P.C. and Famoye, F. (1989) The Truncated Generalized Poisson Distribution and its Estimation. Communications in Statistics: Theory and Methods 18(10), 3635-3648.

Consul, P.C. and MitTal, S.P. (1975) A New Urn Model with Predetermined Strategy. Biometrical Journal 17, 67-75.

Consul, P.C. and MitTal, S.P. (1977) Some Discrete Multinomial Probability Models with Predetermined Strategy. Biometrical Journal 19, 163-176.

Consul, P.C. and Shoukri, M.M. (1985) The Generalized Poisson Distribution When the Sample Mean is Larger than the Sample Variance. Communications in Statistics: Simulation and Computation 14, 667-681.

Famoye, F. and Consul, P.C. (1995) Bivariate Generalized Poisson Distribution with Some Applications. Metrika 42, 127-138.

Famoye, F., Wulu, J.T. and Singh, K.P. (2004) On the Generalized Poisson Regression Model with an Application to Accident Data. Journal of Data Science 2, 287-295.

Gelman, A., Carlin, J.B., Stern, H.S. and Rubin, D.B. (2004) Bayesian Data Analysis, Second edition. Boca Raton: Chapman \& Hall/CRC.

Henze, N. and Klar, B. (1995) Bootstrap Based Goodness of Fit Tests for the Generalized Poisson Model. Communications in Statistics: Theory and Methods 24(7), 1875-1896.

Klugman, S.A., Panjer, H.H. and Willmot, G.E. (2004) Loss Models: From Data to Decisions, Second edition. New York: John Wiley \& Sons, Inc.

KrishnaJi, N. (1974) Characterization of Some Discrete Distributions Based on a Damage Model. Sankhya, Series A 36, 204-213.

Makov, U.E. (2001) Principal Applications of Bayesian Methods in Actuarial Science: A Perspective. North American Actuarial Journal 5(4), 53-73.

Makov, U.E, Smith, A.F.M. and Liu, Y.-H. (1996) Bayesian Methods in Actuarial Science. The Statistician 45(4), 503-515.

Morocoima-Black, R., Chavarra, S. and Lucas, C. (2001) Selected Intersection Crash Analysis for 1993-1998. Champaign-Urbana Urbanized Area Transportation Study (CUUATS), Champaign County Regional Planning Commission. 
Ntzoufras, I. and Dellaportas, P. (2002) Bayesian Modeling of Outstanding Liabilities Incorporating Claim Count Uncertainty (with discussion). North American Actuarial Journal 6(1), 113-128.

Ntzoufras, I., Katsis, A. and Karlis, D. (2005) Bayesian Assessment of the Distribution of Insurance Claim Counts Using Reversible Jump MCMC. North American Actuarial Journal 9(3), 90-108.

Rao, C.R. and Rubin, H. (1964) On a Characterization of the Poisson Distribution. Sankhya, Series A 26, 295-298.

RaO, C.R. (1965) On Discrete Distributions Arising Out of Methods of Ascertainment. Sankhya, Series A 27, 311-324.

RaO, M.B. and Shanbhag, D.N. (1982) Damage Models. Encyclopedia of Statistical Sciences, Volume 2, pages 262-265. Banks, D.L., Read, C.B. and Kotz, S. (editors).

Rosenberg, M. and Young, V.R. (1999) A Bayesian Approach to Understanding Time Series Data. North American Actuarial Journal 3(2), 130-143.

SCollnik, D.P.M. (1995a) Bayesian Analysis of Generalized Poisson Models for Claim Frequency Data Utilising Markov Chain Monte Carlo Methods. Actuarial Research Clearing House 1995.1, 339-356.

Scollnik, D.P.M. (1995b) Bayesian Analysis of Two Overdispersed Poisson Models. Biometrics 51, 1117-1126.

SCollnik, D.P.M. (1998) On the Analysis of the Truncated Generalized Poisson Distribution Using a Bayesian Method. ASTIN Bulletin 28(1), 135-152.

Scollnik, D.P.M. (2001) Actuarial Modeling with MCMC and BUGS. North American Actuarial Journal 5(2), 96-124.

Spiegelhalter, D.J., Best, N.G., Carlin, B.P. and van Der Linde, A. (2002) Bayesian Measures of Model Complexity and Fit. Journal of the Royal Statistical Society, Series B: Statistical Methodology 64, 583-616.

Spiegelhalter, D.J., Thomas, A., Best, N.G. and Gilks, W.R. (1996) BUGS : Bayesian inference Using Gibbs Sampling. MRC Biostatistics Unit, Cambridge.

Spiegelhalter, D.J., Thomas, A., Best, N.G. and Lunn, D. (2004) WinBUGS User Manual: Version 2.0, June 2004. Available at mathstat.helsinki.fi/openbugs.

Srivastava, R.C. and Srivastava, A.B.L. (1970) On a Characterization of Poisson distribution. Journal of Applied Probability 7, 497-501.

Sutton, J., Standen, P. and Wallace, A. (1994) Unreported accidents to patients in hospital. Nursing Times 90 (39), 46-49.

Venables, W.N., Smith, D.M. and the R Development Core Team (2005) An Introduction to R, Notes on R: A Programming Environment for Data Analysis and Graphics, Version 2.1.1 (200506-20). Available at cran.r-project.org/manuals.html.

Vernic, R. (1997) On the Bivariate Generalized Poisson Distribution. ASTIN Bulletin 27, 23-32.

Vernic, R. (2000) A Multivariate Generalization of the Generalized Poisson Distribution. ASTIN Bulletin 30, 57-67.

Verrall, R.J. (2004) A Bayesian Generalized Linear Model for the Bornhuetter-Ferguson Method of Claims Reserving. North American Actuarial Journal 8(3), 67-89.

\author{
DAVID P.M. SCOLLNIK \\ Department of Mathematics and Statistics \\ University of Calgary \\ 2500 University Drive N.W. \\ Calgary, Alberta, Canada T2N $1 N 4$ \\ E-mail:scollnik@math.ucalgary.ca
}

